\title{
Parasitic antigens alter macrophage polarization during Schistosoma japonicum infection in mice
}

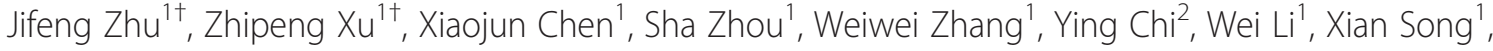 \\ Feng Liu $^{1}$ and Chuan Su${ }^{{ }^{*}}$
}

\begin{abstract}
Background: Schistosome eggs are trapped in host liver and elicit severe hepatic granulomatous inflammation, which can lead to periportal fibrosis, portal hypertension, hemorrhage, or even death in the host. It was reported that the macrophage plays an important role in host immune responses to schistosome infection. Nitric oxide (NO) produced by classically activated macrophages (M1 macrophages) is cytotoxic to schistosomula and can prevent hepatic schistosomal fibrosis, while arginase-1 (Arg-1) expressed by alternatively activated macrophages (M2 macrophages) promotes hepatic schistosomal fibrosis. However, the dynamics of macrophage polarization, as well as the possible factors that regulate macrophage polarization, during schistosome infection remain unclear.

Methods: We first analyzed M1 and M2-phenotypic markers of peritoneal macrophages from mice infected with Schistosoma japonicum (S. japonicum) at indicated time points using flow cytometry (FCM) analysis and real-time PCR. Then we treated peritoneal macrophages from normal mice with schistosome worm antigen (SWA) or schistosome soluble egg antigen (SEA) and determined M1 and M2-phenotypic markers, in order to identify macrophage polarization in responding to schistosomal antigens.
\end{abstract}

Results: In this study, we showed that macrophages were preferentially differentiated into the M1 subtype during the acute stage of S. japonicum infection. However, the level of M1 macrophages decreased and M2 macrophages significantly increased during the chronic stage of infection. Furthermore, we showed that SWA favors the generation of M1 macrophages, whereas SEA preferentially promotes M2-polarized phenotype.

Conclusion: These findings not only reveal the parasite antigen-driven dynamic changes in macrophage polarization, but also suggest that manipulation of macrophage polarization may be of therapeutic benefit in controlling excessive hepatic granulomas and fibrosis in the host with schistosomiasis.

Keywords: Schistosoma japonicum, Liver fibrosis, Macrophage polarization, Schistosomal antigen

\section{Background}

Schistosomiasis is a tropical disease caused by worms of the genus Schistosoma. 200 million people are infected worldwide, leading to the loss of 1.53 million disabilityadjusted life years [1]. During schistosome infection, in the first three weeks post-infection, the schistosomula migrating in the host are shown to be the main target of immune attack [2-5]. Since 4-5 weeks post-infection the parasites begin to produce eggs. The eggs trapped in

\footnotetext{
* Correspondence: chuansu@njmu.edu.cn

${ }^{+}$Equal contributors

'Department of Pathogen Biology and Immunology, Jiangsu Key Laboratory of Pathogen Biology, Nanjing Medical University, 140 Hanzhong Road,

Nanjing, Jiangsu 210029, China

Full list of author information is available at the end of the article
}

host tissues evoke granulomatous responses, which peak around week 8 post-infection, and result in acute symptoms in patients $[2,6]$. During the chronic phase of the infection (since 11-13 weeks post-infection), increasing collagen deposits in the host liver and fibrosis develops [2,6].

Studies have identified macrophages, one of the main cellular constituents of granulomas [6,7], as critical regulators of liver fibrosis [8-10]. Macrophages are known to retain considerable plasticity and can respond to environmental signals by changing effector phenotypes [11,12]. Macrophages can be categorized into two subpopulations: classically activated macrophages (M1 macrophages) and alternatively activated macrophages (M2 macrophages). M1 macrophages that develop in response to pro-inflammatory 
stimuli such as LPS or IFN- $\gamma$ are characterized by the expression of high levels of CD16/32, TNF- $\alpha$, IL-12, inducible nitric oxide synthase (iNOS) and chemokines such as CXCL9, CXCL10 and CXCL11. M2 macrophages, can be induced by IL-4 or chitin, are characterized by their high expression of mannose receptor (Mrc1 encoding MR, also known as CD206), arginase-1 (Arg-1), IL-10 and chemokines such as CCL2, CCL17 and CCL22 [11-15].

During schistosome infection, M1 macrophages are thought to be cytotoxic to schistosomula as M1 macrophages produced nitric oxide (NO), which is an effector mechanism previously implicated in the killing of schistosomula [3]. M2 macrophages are important regulators of fibrosis via Arg-1 activation. Arg-1 hydrolyses $\mathrm{L}$-arginine to urea and L-ornithine. L-ornithine can be further metabolized to the amino acid proline, which is required for collagen production and thus promotes the development of fibrosis in the liver during schistosomiasis $[9,10,16,17]$. Nevertheless, L-arginine can also be oxidized by the M1-related enzyme iNOS to NO, which can restrain hepatic fibrosis $[10,16,17]$. In addition, M2related chemokines CCL17 and CCL22 are also thought to contribute to the fibrotic response as mice deficient in these chemokines develop smaller granulomas and less fibrosis [18]. Therefore, it is suggested that interventional control of liver fibrosis by regulating macrophage polarization could be a potential therapeutic strategy for schistosomiasis management.

Up to now, the dynamics of macrophage polarization during schistosome infection have not yet been reported. In addition, the possible role of schistosomal antigens in macrophage polarization remains unclear. The present study investigated the kinetics of macrophage polarization in S. japonicum infected mice and evaluated the possible role of schistosomal antigens in macrophage polarization, which might provide clues to better control of fibrosis in schistosomiasis.

\section{Methods}

\section{Ethics statement}

Animal experiments were performed in strict accordance with the Regulations for the Administration of Affairs Concerning Experimental Animals (1988.11.1), and efforts were made to minimize suffering. Ethical approval was obtained from the Institutional Animal Care and Use Committee (IACUC) of Nanjing Medical University for the use of laboratory animals.

\section{Mice infection}

Six-week-old female CD-1 mice were purchased from SLAC Laboratory Animal Co. Ltd. (Shanghai, China). Each mouse was infected percutaneously with $12 \mathrm{~S}$. japonicum cercariae (Chinese mainland strain). Oncomelania hupensis harboring S. japonicum cercariae were purchased from the
Parasitic Disease Prevention and Research Institute of Jiangsu Province.

\section{Peritoneal macrophage purification}

Peritoneal macrophages were isolated from CD-1 mice as previously described [19]. Briefly, S. japonicum infected or normal control mice were sacrificed at $0,3,8$, or 13 weeks post-infection. Peritoneal exudate cells (PECs) were isolated by peritoneal lavage using PBS with $1 \%$ FBS. PECs were obtained by centrifugation at $500 \mathrm{~g}$ for $5 \mathrm{~min}$ at $4^{\circ} \mathrm{C}$ and resuspended in ACK lysis buffer $(0.15 \mathrm{M}$ $\mathrm{NH}_{4} \mathrm{Cl}, 10 \mathrm{mM} \mathrm{KHCO}, 0.1 \mathrm{mM} \mathrm{Na} 2 \mathrm{EDTA}, \mathrm{pH}$ 7.2) for erythrocyte lysis. Subsequently, cells were centrifuged at $500 \mathrm{~g}$ for $5 \mathrm{~min}$ at $4^{\circ} \mathrm{C}$ and finally resuspended in DMEM medium supplemented with $10 \%$ FBS, $1 \%$ penicillinstreptomycin. PECs were cultured in 6-well plates (Costar, Cambridge, MA) at a density of $5 \times 10^{6} /$ well to allow macrophages to adhere. Macrophages were purified by adherence for $2.5 \mathrm{~h}$, at which time media were replaced to remove non-adherent cell. Adherent macrophages were incubated with $5 \mathrm{mM}$ EDTA/PBS for $10 \mathrm{~min}$ at $37^{\circ} \mathrm{C}$, and then detached by vigorous pipetting to prepare single-cell suspension for purity analysis. As determined by flow cytometry (FCM) analysis using PE-conjugated antibody against mouse $\mathrm{F} 4 / 80$ and $\mathrm{APC}$-conjugated antibody against mouse CD11b (eBioscience, San Diego, CA), the purity of isolated macrophage was $>98 \%$.

\section{In vitro treatment of peritoneal macrophage}

Peritoneal macrophages were purified from normal mice as described above. For antigen treatment, macrophages were washed with PBS to remove the non-adherent cells. Then PBS, $100 \mu \mathrm{g} / \mathrm{ml}$ SWA, $10 \mu \mathrm{g} / \mathrm{ml}$ SEA, $1 \mu \mathrm{g} / \mathrm{ml} \mathrm{LPS}$ (Sigma, St. Louis, MO) or $1000 \mathrm{U} / \mathrm{ml} \mathrm{IL-4} \mathrm{(Peprotech}$ Inc., Rocky Hill, NJ) were added directly to each well with fresh medium, respectively. Cells were then incubated for $24 \mathrm{~h}$ at $37^{\circ} \mathrm{C}$. Macrophages treated with LPS or IL-4 was used as positive controls for M1 and M2 macrophages, respectively [14].

\section{Flow cytometry}

Single-cell suspensions were washed in PBS with $1 \%$ FBS and adjusted to $1 \times 10^{6}$ cells per $100 \mu \mathrm{l}$ PBS with $1 \%$ FBS. For purity analysis of macrophage, cells were incubated with PE-conjugated antibody against mouse F4/80 and $\mathrm{APC}$-conjugated antibody against mouse $\mathrm{CD} 11 \mathrm{~b}$ (eBioscience). For M1 and M2 surface marker analysis, cells were incubated with PE-conjugated antibodies against mouse CD16/32 or CD206 (eBioscience). PE- or APC- conjugated rat IgG2a (eBioscience) served as control antibodies. All antibodies were used at $5 \mu \mathrm{g} / \mathrm{ml}$. The cells were incubated with the antibodies for $30 \mathrm{~min}$ at $4{ }^{\circ} \mathrm{C}$ and washed with PBS. The samples were fixed with $1 \%$ paraformaldehyde/PBS and analyzed by using a BD FACSCalibur 
Flow Cytometer. Results were analyzed using FlowJo software (Tree star Inc, Ashland, OR).

\section{Real-time PCR}

Macrophage total RNA was isolated using TRIzol (Invitrogen, Carlsbad, CA), converted to cDNA using a Reverse Transcription kit (Fermantas Life Sciences, St. Leon-Rot, Baden-Württemberg, Germany) as the manufacturer's instructions. Quantitative real-time PCR was conducted using FastStart Universal SYBR Green Master (Rox) reagents (Roche Diagnostics, Indianapolis, IN) and a 7300 Real-time PCR machine (Applied Biosystems, Foster City, CA). The reaction conditions consisted of: stage $1,50^{\circ} \mathrm{C}$ for $2 \mathrm{~min}$; stage $2,95^{\circ} \mathrm{C}$ for $10 \mathrm{~min}$; stage 3,40 cycles of $95^{\circ} \mathrm{C}$ for $15 \mathrm{~s}$ and $60^{\circ} \mathrm{C}$ for $1 \mathrm{~min}$, which were concluded by the melting curve analysis process. Fold changes of gene expression were calculated using the $2^{-\Delta \Delta \mathrm{Ct}}$ method. The sequences of the primer pairs used in this analysis are as follows: Tnf: forward, $5^{\prime}$-catcttctcaaaattcgagtgacaa-3', reverse, 5 '-tgggagtagacaaggtacaaccc-3'; Il12a: forward, 5' gacagtffaggcaccaggcc- 3 ', reverse, $5^{\prime}$-cagacatcgctgtcccggcg3'; Il10: forward, 5' -actttaagggttacttgggttgc-3', reverse, 5'-atttcacaggggagaaatcg-3'; Cxcl9: forward, 5'-tctcggactt cactccaacaca- $3^{\prime}$, reverse, $5^{\prime}$-actccacactgctggaggaaga- $3^{\prime}$; Cxcl10: forward, 5' -ccgtcattttctgcctcatcc-3', reverse, 5'ccctatggccctcattctca-3'; Cxcl11: forward, 5' -gaacaggaaggt cacagccatagc-3', reverse, 5' -tcaactttgtcgcagccgttactc-3'; Ccl2: forward, $5^{\prime}$-aagccagctctctcttcctcca-3', reverse, $5^{\prime}$ aagccagctctctcttcctcca-3'; Ccl17: forward, 5'-agtgctgcctgg attacttcaaag- $3^{\prime}$, reverse, $5^{\prime}$-ctggacagtcagaaacacgatgg- $3^{\prime}$;

A

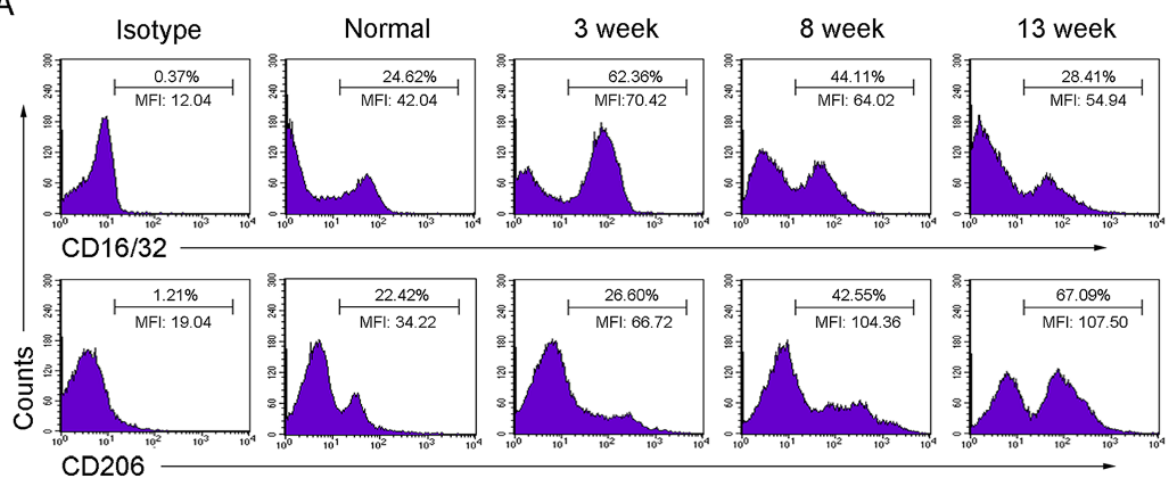

B
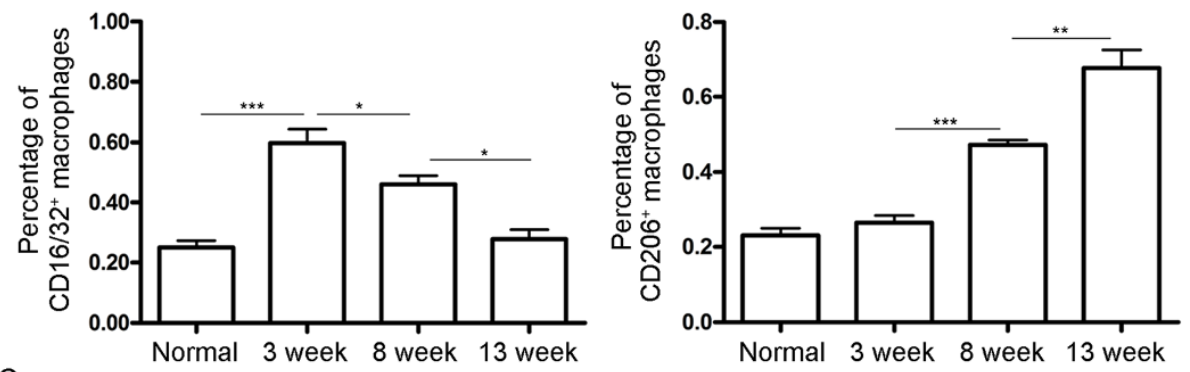

C
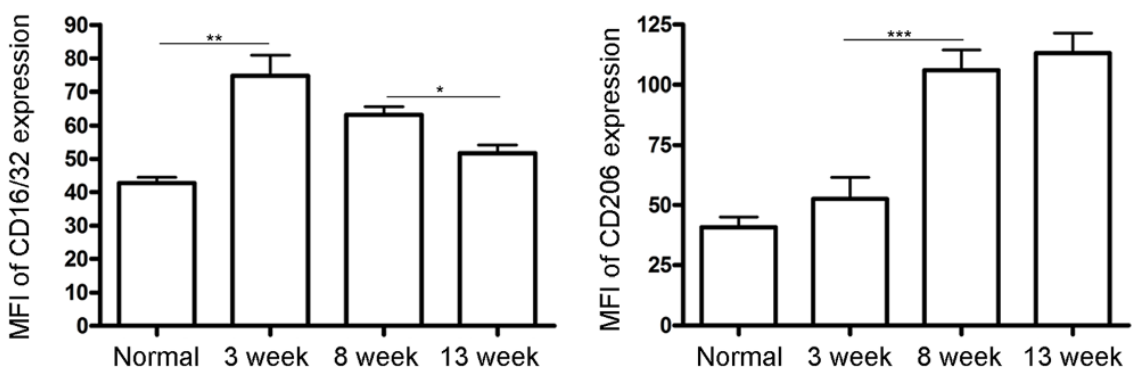

Figure 1 Changes in percentages of M1 and M2 macrophages during S. japonicum infection. Peritoneal macrophages were purified from normal or S. japonicum infected mice at indicated time points post-infection. Expression of CD16/32 (M1) and CD206 (M2) were evaluated by FCM analysis. (A) Representative histograms obtained by FCM analysis. (B) Percentages of CD16/32 $2^{+}$macrophages and CD206 ${ }^{+}$macrophages. (C) Mean fluorescence intensity (MFI) of CD16/32 and CD206 expression on macrophages. Data are expressed as the mean \pm SD of three independent experiments with 6 mice per group in each experiment. 
Ccl22: forward, $5^{\prime}$-taacatcatggctaccctgcg-3', reverse, $5^{\prime}$ tgtcttccacattggcacca-3'; Nos2: forward, 5'-gccaccaacaatggc aaca-3', reverse, 5' - cgtaccggatgagctgtgaatt-3'; Arg1: forward, $5^{\prime}$-cagaagaatggaagagtcag- $3^{\prime}$, reverse, 5' -cagatatgcagg gagtcacc-3'; Irf5: forward, 5' -aataccccaccaccttttga-3', reverse, 5'-aataccccaccacctttga-3'; Gapdh: forward, 5' -ggtgaaggtcggt gtgaacg-3', reverse, $5^{\prime}$-accatgtagttgaggtcaatgaagg-3'.

\section{Preparation of SWA and SEA}

For SWA preparation, S. japonicum adult worms were suspended in cold diethyl ether and homogenized on ice using a homogenizer (VirTis Co., Inc., Gardiner, NY). The homogenate was centrifuged at $2,000 \mathrm{~g}$ for $5 \mathrm{~min}$ at $4^{\circ} \mathrm{C}$ to remove lipids. The pellet was freeze-thawed several times in PBS mixed with $1 \mathrm{mM}$ PMSF (Roche Diagnostics) and $2 \mathrm{ug} / \mathrm{ml}$ Leupeptin (Sigma). The homogenate was centrifuged at 20,000 g for $50 \mathrm{~min}$ at $4^{\circ} \mathrm{C}$ and the supernatant was filtered through $0.22 \mu \mathrm{m}$ filter (Millipore Corporation). Protein concentration of worm extracts were determined by BCA Protein Assay Kit (Bio-Rad) and the extracts were stored at $-80^{\circ} \mathrm{C}$ before use [20].

To prepare SEA, $S$. japonicum eggs were suspended in PBS containing $1 \mathrm{mM}$ PMSF (Roche Diagnostics) and $2 \mu \mathrm{g} / \mathrm{ml}$ Leupeptin (Sigma) and homogenized on ice using a homogenizer (VirTis Co.). The suspension was freezethawed several times and centrifuged at 20,000 $\mathrm{g}$ for $50 \mathrm{~min}$ at $4^{\circ} \mathrm{C}$. The supernatant was filtered through
$0.22 \mu \mathrm{m}$ filter (Millipore Corporation). Protein concentration of egg extracts were determined by BCA Protein Assay Kit (Bio-Rad) and the extracts were stored at $-80^{\circ} \mathrm{C}$ before use [21].

\section{Statistical analysis}

Results are shown as the mean \pm standard deviation (SD) for at least 3 repeated independent experiments for each group. Statistical comparisons were determined by using SPSS and Student's t test for independent samples. Significant differences were as follows: ${ }^{*} \mathrm{P}<0.05$; ${ }^{* *} \mathrm{P}<0.005$; ${ }^{* * *} \mathrm{P}<0.001$.

\section{Results}

\section{Changes in percentages of M1 and M2 macrophages} during $S$. japonicum infection

As shown in Figure $1 \mathrm{~A}$ and $1 \mathrm{~B}$, the percentage of M1 macrophages significantly increased 3 weeks after infection but decreased quickly by 8 weeks post-infection. However, the increase in the percentage of M2 macrophages started at 8 weeks after infection. Similar results were obtained in mean fluorescence intensity (MFI) of CD16/32 and CD206 expression, which reflects the average level of CD16/32 and CD206 expressed on a single M1 or M2 macrophage cell, respectively (Figure $1 \mathrm{~A}$ and $1 \mathrm{C}$ ). These results suggest that macrophages are typically skewed from the M1 phenotype at the acute stage of S. japonicum infection toward the M2 phenotype at the chronic stage.

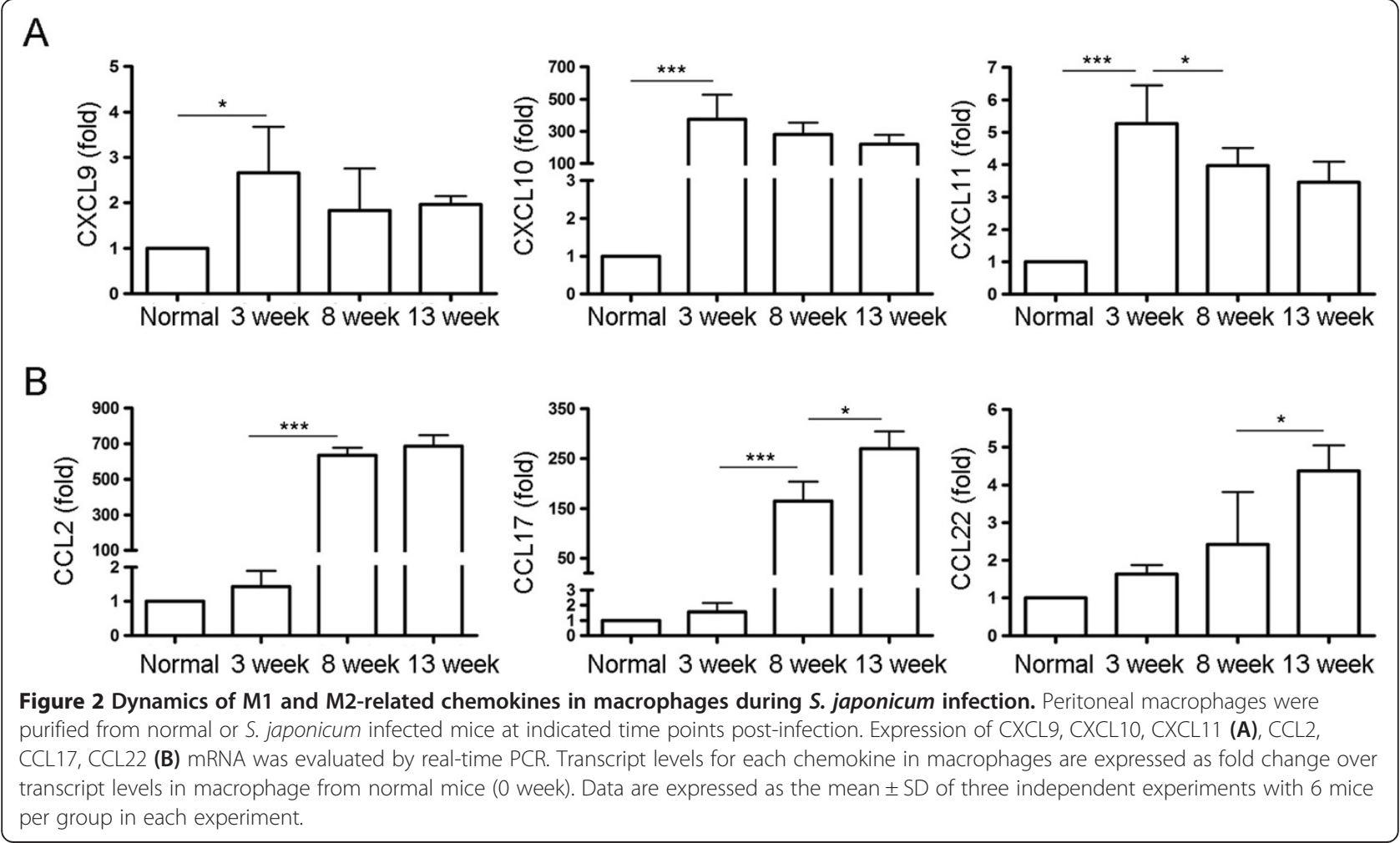



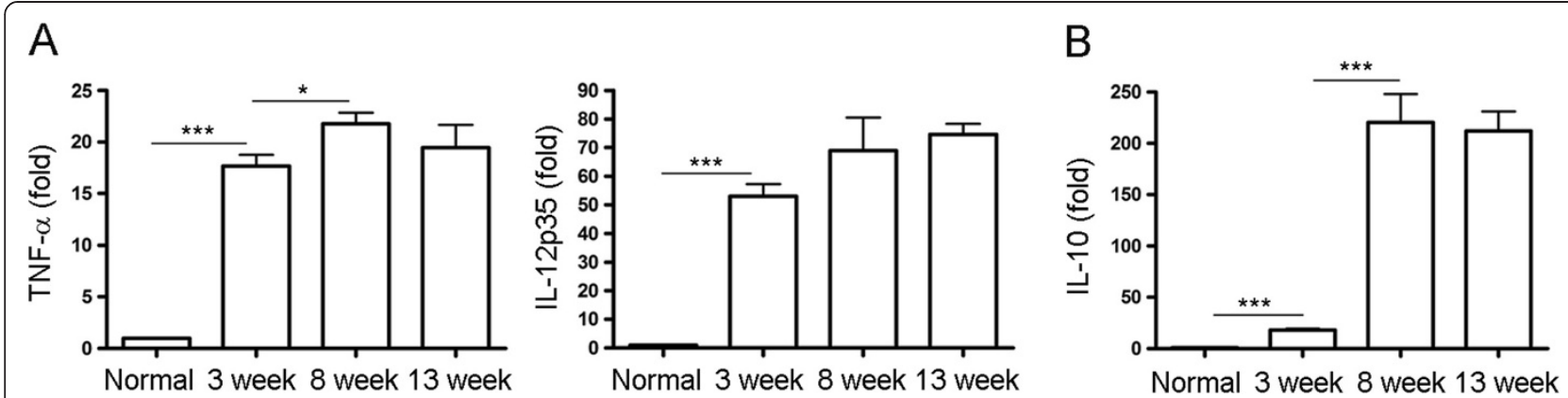

Figure 3 Dynamics of M1 and M2-related cytokine expression in macrophages during S. japonicum infection. Peritoneal macrophages were purified from normal or S. japonicum infected mice at indicated time points post-infection. Expression of TNF-a, IL-12p35 (A), IL-10 (B) mRNA was evaluated by real-time PCR. Transcript levels for each cytokine in macrophages are expressed as fold change over transcript levels in macrophage from normal mice (0 week). Data are expressed as the mean \pm SD of three independent experiments with 6 mice per group in each experiment.

\section{Dynamics of M1 and M2-related chemokines in macrophages during S. japonicum infection}

The kinetics of M1 and M2 chemokine mRNA expression in peritoneal macrophages of $S$. japonicum infected mice were assessed by real-time PCR. As shown in Figure 2, M1-related chemokines CXCL9, CXCL10, and CXCL11 significantly increased after 3 weeks of infection and then declined. However, M2-related chemokines CCL2, CCL17, and CCL22 progressively increased during the period of 13 weeks infection.

\section{Dynamics of M1 and M2-related cytokine expression in macrophages during S. japonicum infection}

M1 and M2 cytokine mRNA expressed by peritoneal macrophages of $S$. japonicum infected mice was detected by real-time PCR. Result in Figure 3A showed that 3 weeks after infection, TNF- $\alpha$ and IL-12mRNAs were significantly increased. A further, but slight increase in the expression of both cytokines was observed 8 weeks after infection and the levels of both cytokines were not changed much at 13 weeks. However, a remarkable increase in the expression of IL-10 was observed 8 weeks after infection and maintained at a high level at 13 weeks (Figure 3B).

\section{Dynamics of M1 and M2-specific enzyme expression in macrophages during $S$. japonicum infection}

As shown in Figure 4A, a significant increase of iNOS, an M1-specific enzyme in macrophages, was observed after 3 weeks of infection, while it was significantly decreased by 8 weeks after infection. In contrast, Arg-1, an M2-specific enzyme in macrophages, showed a slight decrease at week 3 after infection, followed by a marked upregulation at week 8 after infection (Figure 4B).

\section{Differential effects of SWA and SEA on macrophage polarization}

To investigate whether M1 and M2 macrophages were induced by antigens derived from different stages of the schistosomal life cycle, peritoneal macrophages from normal mice were purified and treated with SWA or SEA in vitro. Result showed that compared to PBS control, SWA stimulation significantly increased the percentage of M1 but not M2 macrophages. However, compared to PBS control, SEA stimulation significantly increased the percentage of M2 but not M1 macrophages (Figure 5A). Results in Figure 5B, 5C and 5D showed that SWA stimulation preferentially enhanced the expression of the
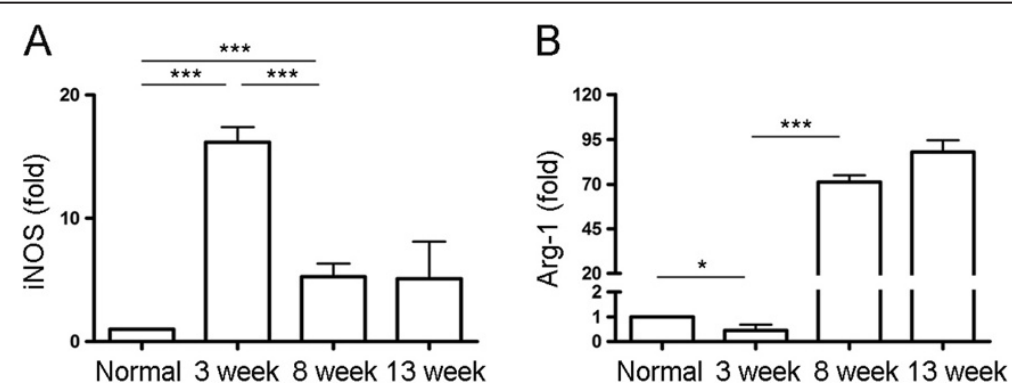

Figure 4 Dynamics of M1 and M2-specific enzyme expression in macrophages during S. japonicum infection. Peritoneal macrophages were purified from normal or S. japonicum infected mice at indicated time points. Expression of iNOS (A) and Arg-1 (B) mRNA was evaluated by real-time PCR. Transcript levels for each enzyme in macrophages are expressed as fold change over transcript levels in macrophage from normal mice ( 0 week). Data are expressed as the mean \pm SD of three independent experiments with 6 mice per group in each experiment. 
A
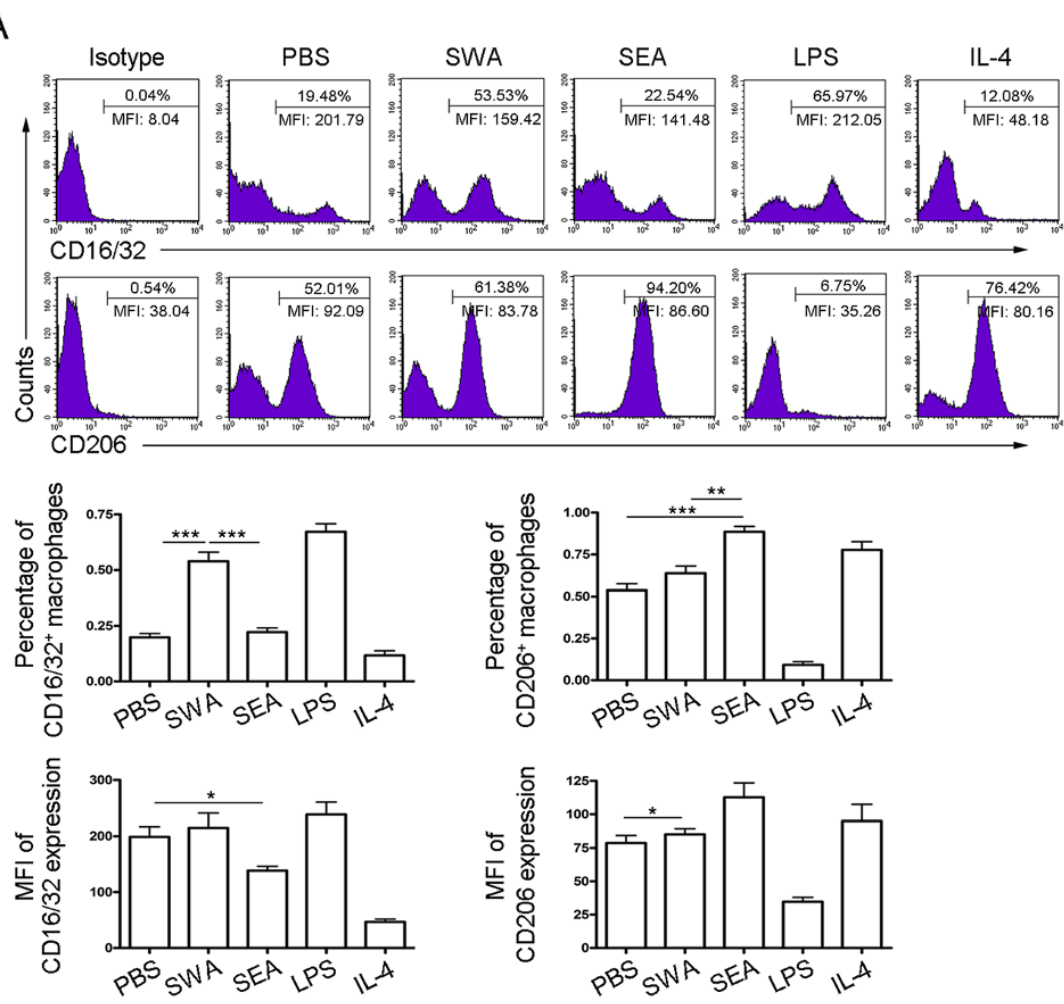

B
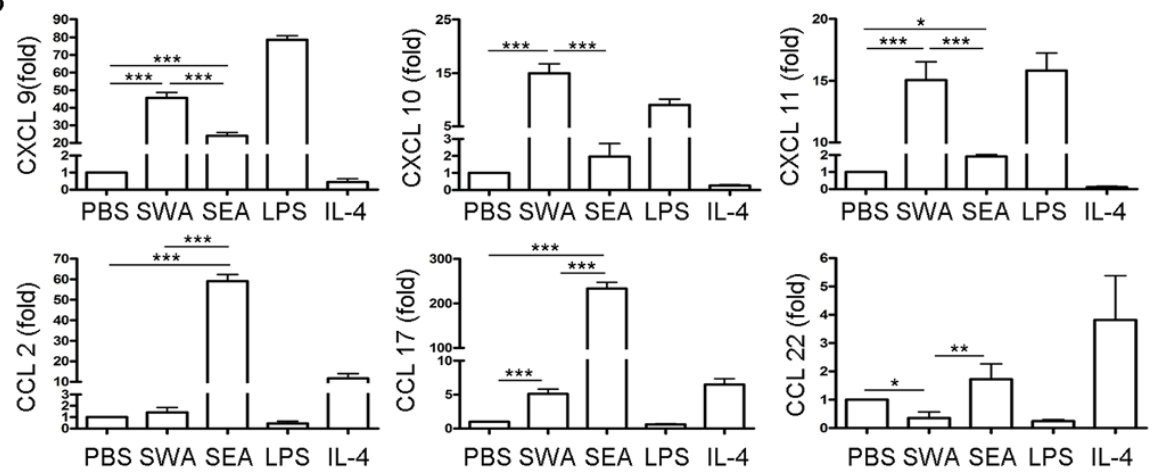

C
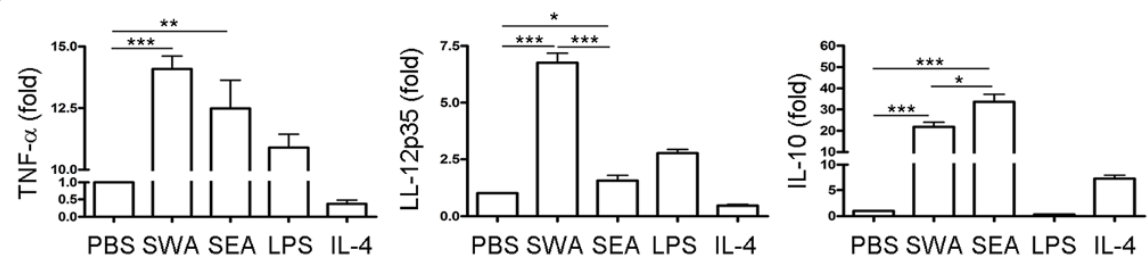

D
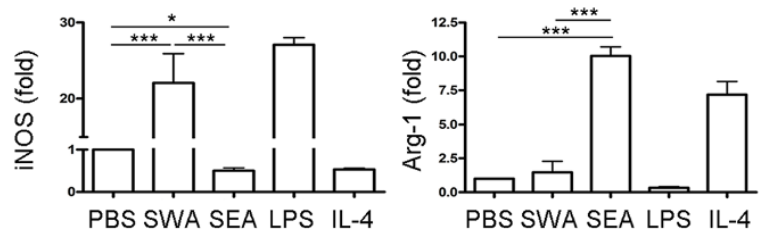

Figure $\mathbf{5}$ (See legend on next page.) 
(See figure on previous page.)

Figure 5 Differential effects of SWA and SEA on macrophage polarization. Peritoneal macrophages from normal mice were purified and treated as described in Methods. LPS was included as positive controls for classical activation and IL-4 for alternative activation. The expression of CD16/32 and CD206 were evaluated by FCM analysis (A). Representative histograms obtained by FCM analysis (top row). Percentages of CD16/32+ macrophages and CD206 ${ }^{+}$macrophages (middle row). Mean fluorescence intensity (MFI) of CD16/32 and CD206 expression on macrophages (bottom row). The transcript levels for chemokines (B), cytokines (C) and enzymes of arginine metabolism (D) were evaluated by real-time PCR. The expression levels for each molecule were normalized to the level in macrophages treated with PBS. Data were mean \pm SD of three independent experiments.

M1-related chemokines (CXCL9, CXCL10 and CXCL11), cytokines (TNF- $\alpha$ and IL-12), and key enzyme of arginine metabolism (iNOS) in macrophages. In contrast, SEA stimulation preferentially enhanced the expression of the M2-related chemokines (CCL2, CCL17 and CCL22), cytokine (IL-10), and key enzyme of arginine metabolism (Arg-1) in macrophages. Together, these data suggest that during schistosome infection, worm antigens may preferentially elicit pro-inflammatory M1 profile of macrophages, while egg antigens may preferentially induce anti-inflammatory M2 phenotype and downregulate the M1 phenotype.

\section{Discussion}

Schistosomiasis continues to be a significant cause of parasitic morbidity and mortality worldwide [1]. Schistosome egg-induced hepatic granuloma formation can lead to tissue destruction and fibrosis, which causes much of the morbidity and mortality associated with this disease. Macrophages are critical for granuloma formation and the development of hepatic fibrosis during schistosomiasis $[2,6]$. In this study, we showed that in response to schistosomal antigens, macrophages were typically skewed from M1 towards the M2 polarization during S. japonicum infection.

Macrophages are functionally polarized into M1 and M2 phenotypes in response to microorganism infection and host mediators [11-15]. Studies suggest that M1 macrophages are favorable to kill schistosomula by producing NO [3], which also plays a role in preventing hepatic fibrosis [16]. In contrast, M2 macrophages are thought to contribute to schistosome-induced fibrosis via the metabolism of 1-arginine to proline and polyamine by Arg-1 $[9,10,16,17]$. However, factors such as Jmjd3-IRF4 axis involved in the mechanism of M2 polarization have been shown to inhibit M1 polarization and the production of NO by M1 macrophages [12]. Therefore, M2 macrophages may promote the survival of parasites. Considering the peritoneal cavity is suggested by studies as a secondary site of schistosome-induced inflammation [22,23], and the phenotype analysis of peritoneal macrophages is technically easier and widely used in studies of the responses of macrophages related to hepatic injuries such as schistosomiasis, cirrhosis and LPS-induced hepatitis [22,24,25], we analyzed the dynamics of peritoneal macrophage polarization during schistosome infection. Our results showed that a predominant M1 phenotype in peritoneal macrophages was observed in the first 3 weeks post-infection, a period when schistosomula migrate from the skin to the hepatic portal system [2]. These immature worms in migration are reported to be susceptible to activated macrophagemediated cytotoxicity $[3,4,26]$. In addition, our results showed that a pronounced M2 activation profile was displayed in peritoneal macrophages at 8 weeks postinfection, a time-point when the severity of hepatic granulomatous response reaches its peak and the fibrosis starts to develop quickly $[2,6]$.

Although we have characterized the dynamics of peritoneal macrophage polarization during schistosome infection, the possible role of schistosomal antigens in macrophage polarization is still unclear. SWA and SEA are two major schistosome antigen mixtures [27-29]. Our results demonstrated that SWA or SEA preferentially induced the macrophages to differentiate to M1- or M2-type cells, respectively. Consistently, recently work demonstrated that hemozoin, a substance isolated from S. mansoni, prevented IL-4-inducted alternative activation of bone marrow-derived macrophages in an in vitro induction system [30]. M1 macrophages are considered to be pro-inflammatory, whereas M2 macrophages are antiinflammatory and limit the tissue-damaging activity of M1 macrophages [13,31,32]. Thus, when the host immunity including M1 response cannot eradicate persistent schistosome infection but has to limit collateral severe tissue damage, the switch of macrophage function from killing to repair, which occurs in response to schistosomal antigens, is likely beneficial to the host. Indeed, some other pathogens, such as Helicobacter pylori [33,34] and Trypanosoma cruzi [35,36], have been shown to induce M2 macrophages to escape killing by M1 macrophages. Therefore, our results also suggested modulation of macrophage polarization by schistosomal antigens as a potential mechanism for both the immune escape of the parasite and the survival strategy of the host during the schistosome infection. Although we have demonstrated the direct role of SWA and SEA in macrophage polarization, previous study also suggest that levels of Th1 and Th2 cytokines, which are affected by SWA and SEA [37-39], are able to modulate M1/M2 skewing [12,40]. Thus, we currently still cannot rule out the possibility that the shift of the M1/M2 polarization may partially 
through the indirect effect of the Th1/Th2 shift during $S$. japonicum infection.

\section{Conclusions}

In summary, our study described the dynamic changes of macrophage polarization, from M1 to M2 subtypes, during S. japonicum infection and revealed distinct roles for schistosomal antigens in regulating macrophage polarization. Our findings not only suggest that signals (antigens) from specific stage of parasite life cycle can skew phenotypic and functional differentiation of macrophages which benefits the parasite and host, but also suggest that these antigens may be potential therapeutic targets for the control of the schistosomal fibrosis.

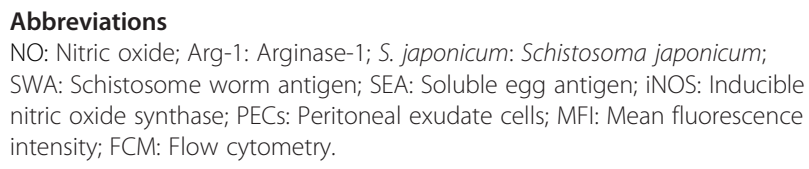

\section{Competing interests}

The authors declare that they have no competing interests.

\section{Authors' contributions}

CS conceived and designed the experiments. JZ and ZX analyzed the data. ZX, XC, SZ, WZ, YC, WL, XS, FL performed the experiments. Manuscript was written by CS and JZ. All authors read and approved the final manuscript.

\section{Acknowledgment}

This work was supported by the grant from the National Natural Science Foundation of China (No. 81271861 and No. 81071385) and the grants from Jiangsu Province (12KJA310001) to Chuan Su. In addition, this is a project partially funded by the Priority Academic Program Development of Jiangsu Higher Education Institutions (PAPD) and Nanjing Medical University (JX21831802/005).

\section{Author details}

${ }^{1}$ Department of Pathogen Biology and Immunology, Jiangsu Key Laboratory of Pathogen Biology, Nanjing Medical University, 140 Hanzhong Road, Nanjing, Jiangsu 210029, China. ${ }^{2}$ Key Laboratory of Enteric Pathogenic Microbiology, Ministry of Health, Institute of Pathogenic Microbiology, Jiangsu Provincial Center for Disease Prevention and Control, Nanjing, Jiangsu 210009, China.

Received: 20 January 2014 Accepted: 11 March 2014 Published: 25 March 2014

\section{References}

1. Gryseels B, Polman K, Clerinx J, Kestens L: Human schistosomiasis. Lancet 2006, 368:1106-1118.

2. Burke ML, Jones MK, Gobert GN, Li YS, Ellis MK, McManus DP: Immunopathogenesis of human schistosomiasis. Parasite Immunol 2009, 31:163-176.

3. Ahmed SF, Oswald IP, Caspar P, Hieny S, Keefer L, Sher A, James SL: Developmental differences determine larval susceptibility to nitric oxide-mediated killing in a murine model of vaccination against Schistosoma mansoni. Infect Immun 1997, 65:219-226.

4. Pearce EJ, James SL: Post lung-stage schistosomula of Schistosoma mansoni exhibit transient susceptibility to macrophage-mediated cytotoxicity in vitro that may relate to late phase killing in vivo. Parasite Immunol 1986, 8:513-527.

5. Burke ML, McGarvey L, McSorley HJ, Bielefeldt-Ohmann H, McManus DP, Gobert GN: Migrating Schistosoma japonicum schistosomula induce an innate immune response and wound healing in the murine lung. Mol Immunol 2011, 49:191-200.
6. Pearce EJ, MacDonald AS: The immunobiology of schistosomiasis. Nat Rev Immunol 2002, 2:499-511.

7. Hernandez HJ, Wang Y, Tzellas N, Stadecker MJ: Expression of class II, but not class I, major histocompatibility complex molecules is required for granuloma formation in infection with Schistosoma mansoni. Eur J Immunol 1997, 27:1170-1176.

8. Wynn TA, Barron L: Macrophages: master regulators of inflammation and fibrosis. Semin Liver Dis 2010, 30:245-257.

9. Barron L, Wynn TA: Macrophage activation governs schistosomiasis-induced inflammation and fibrosis. Eur J Immunol 2011, 41:2509-2514.

10. Noel W, Raes G, Hassanzadeh Ghassabeh G, De Baetselier P, Beschin A: Alternatively activated macrophages during parasite infections. Trends Parasitol 2004, 20:126-133.

11. Mosser DM, Edwards JP: Exploring the full spectrum of macrophage activation. Nat Rev Immunol 2008, 8:958-969.

12. Sica A, Mantovani $A$ : Macrophage plasticity and polarization: in vivo veritas. J Clin Invest 2012, 122:787-795.

13. Krausgruber T, Blazek K, Smallie T, Alzabin S, Lockstone H, Sahgal N, Hussell T, Feldmann M, Udalova IA: IRF5 promotes inflammatory macrophage polarization and TH1-TH17 responses. Nat Immunol 2011, 12:231-238.

14. Satoh T, Takeuchi O, Vandenbon A, Yasuda K, Tanaka Y, Kumagai Y, Miyake T, Matsushita K, Okazaki T, Saitoh T, Honma K, Matsuyama T, Yui K, Tsujimura T, Standley DM, Nakanishi K, Nakai K, Akira S: The Jmjd3-Irf4 axis regulates M2 macrophage polarization and host responses against helminth infection. Nat Immunol 2010, 11:936-944.

15. Mantovani A, Sica A, Sozzani S, Allavena P, Vecchi A, Locati M: The chemokine system in diverse forms of macrophage activation and polarization. Trends Immunol 2004, 25:677-686.

16. Hesse M, Modolell M, La Flamme AC, Schito M, Fuentes JM, Cheever AW, Pearce EJ, Wynn TA: Differential regulation of nitric oxide synthase-2 and arginase- 1 by type 1/type 2 cytokines in vivo: granulomatous pathology is shaped by the pattern of I-arginine metabolism. J Immunol 2001, 167:6533-6544.

17. Bronte $\mathrm{V}$, Zanovello P: Regulation of immune responses by L-arginine metabolism. Nat Rev Immunol 2005, 5:641-654.

18. Jakubzick C, Wen H, Matsukawa A, Keller M, Kunkel SL, Hogaboam CM: Role of CCR4 Ligands, CCL17 and CCL22, During Schistosoma mansoni Egg-Induced Pulmonary Granuloma Formation in Mice. Am J Pathol 2004, 165:1211-1221.

19. Wu Y, Li Y-Y, Matsushima K, Baba T, Mukaida N: CCL3-CCR5 Axis regulates intratumoral accumulation of leukocytes and fibroblasts and promotes angiogenesis in murine lung metastasis process. J Immunol 2008, 181:6384-6393.

20. Boamah D, Kikuchi M, Huy NT, Okamoto K, Chen H, Ayi I, Boakye DA, Bosompem KM, Hirayama K: Immunoproteomics identification of major lgE and IgG4 reactive schistosoma japonicum adult worm antigens using chronically infected human plasma. Trop Med Health 2012, 40:89-102.

21. Mandalasi M, Dorabawila N, Smith DF, Heimburg-Molinaro J, Cummings RD, Nyame AK: Development and characterization of a specific lgG monoclonal antibody toward the Lewis $x$ antigen using splenocytes of Schistosoma mansoni-infected mice. Glycobiology 2013, 23:877-892.

22. Abdallahi OMS, Bensalem H, Augier R, Daigana M, De Reggi M, Gharib B: Arginase expression in peritoneal macrophages and increase in circulating polyamine levels in mice infected with Schistosoma mansoni. CMLS, Cell Mol Life Sci 2001, 58:1350-1357.

23. Tanabe M, Kaneko N, Takeuchi T: Schistosoma mansoni: suppression of carbamoyl phosphate synthetase (ammonia) and ornithine carbamoyltransferase activities in the liver of infected mice. Exp Parasitol 1989, 68:432-442.

24. Tapia-Abellán A, Ruiz-Alcaraz AJ, Hernández-Caselles T, Such J, Francés R, García-Peñarrubia P, Martínez-Esparza M: Role of MAP Kinases and PI3K-Akt on the cytokine inflammatory profile of peritoneal macrophages from the ascites of cirrhotic patients. Liver Int 2013, 33:552-560.

25. Jiang W, Sun R, Wei H, Tian Z: Toll-like receptor 3 ligand attenuates LPS-induced liver injury by down-regulation of toll-like receptor 4 expression on macrophages. Proc Natl Acad Sci U S A 2005, 102:17077-17082.

26. James SL, Sher A, Lazdins JK, Meltzer MS: Macrophages as effector cells of protective immunity in murine schistosomiasis. II. Killing of newly transformed schistosomula in vitro by macrophages activated as a consequence of Schistosoma mansoni infection. J Immunol 1982, 128:1535-1540. 
27. Asahi H, Stadecker MJ: Analysis of egg antigens inducing hepatic lesions in schistosome infection. Parasitol Int 2003, 52:361-367.

28. de Jesus Ribeiro A, Araújo I, Bacellar O, Magalhães A, Pearce E, Harn D, Strand M, Carvalho EM: Human immune responses to schistosoma mansoni vaccine candidate antigens. Infect Immun 2000, 68:2797-2803.

29. Abdel-Hafeez EH, Kikuchi M, Watanabe K, Ito T, Yu C, Chen H, Nara T, Arakawa T, Aoki Y, Hirayama K: Proteome approach for identification of schistosomiasis japonica vaccine candidate antigen. Parasitol Int 2009, 58:36-44.

30. Truscott M, Evans DA, Gunn M, Hoffmann KF: Schistosoma mansoni hemozoin modulates alternative activation of macrophages via specific suppression of retnla expression and secretion. Infect Immun 2013, 81:133-142.

31. Fleetwood AJ, Lawrence T, Hamilton JA, Cook AD: Granulocyte-macrophage colony-stimulating factor (CSF) and macrophage CSF-dependent macrophage phenotypes display differences in cytokine profiles and transcription factor activities: implications for CSF blockade in inflammation. J Immunol 2007, 178:5245-5252.

32. Verreck FAW, de Boer T, Langenberg DML, Hoeve MA, Kramer M, Vaisberg E, Kastelein R, Kolk A, de Waal-Malefyt R, Ottenhoff THM: Human IL-23-producing type 1 macrophages promote but IL-10-producing type 2 macrophages subvert immunity to (myco)bacteria. Proc Natl Acad Sci U S A 2004, 101:4560-4565.

33. Chaturvedi R, Asim M, Lewis ND, Algood HMS, Cover TL, Kim PY, Wilson KT: I-arginine availability regulates inducible nitric oxide synthase-dependent host defense against helicobacter pylori. Infect Immun 2007, 75:4305-4315.

34. Fehlings M, Drobbe L, Moos V, Renner Viveros P, Hagen J, Beigier-Bompadre M, Pang E, Belogolova E, Churin Y, Schneider T, Meyer TF, Toni A, Ralf I: Comparative analysis of the interaction of helicobacter pylori with human dendritic cells, macrophages, and monocytes. Infect Immun 2012, 80:2724-2734

35. Cuervo $H$, Pineda MA, Aoki MP, Gea S, Fresno $M$, Gironès $N$ : Inducible nitric oxide synthase and arginase expression in heart tissue during acute trypanosoma cruzi infection in mice: arginase $I$ is expressed in infiltrating CD68+ macrophages. J Infect Dis 2008, 197:1772-1782.

36. Garrido W, Dulgerian LR, Stempin CC, Cerban FM: The increase in mannose receptor recycling favors arginase induction and Trypanosoma cruzi survival in macrophages. Int J Biol Sci 2011, 7:1257-1272.

37. Mountford AP, Wilson RA: Fractionation of schistosome antigens by high performance electrophoretic chromatography and their screening for the ability to induce Th1 lymphocyte activity. I Immunol Methods 1993, 160:237-244.

38. Everts B, Hussaarts L, Driessen NN, Meevissen MH, Schramm G, van der Ham A, van der Hoeven B, Scholzen T, Burgdorf S, Mohrs M, Pearce EJ, Hokke CH, Haas H, Smits HH, Yazdanbakhsh M: Schistosome-derived omega-1 drives Th2 polarization by suppressing protein synthesis following internalization by the mannose receptor. J Exp Med 2012, 209:1753-1767. S1751.

39. Li J, Zhuang W, Cong L, Shi W, Cai X, Huang F, Liao Y, Liu Y, Li J, Chen C, Chen X-P: Cyclophilin A from Schistosoma japonicum promotes a Th2 response in mice. Parasit Vectors 2013, 6:330.

40. Gordon S: Alternative activation of macrophages. Nat Rev Immunol 2003, 3:23-35.

doi:10.1186/1756-3305-7-122

Cite this article as: Zhu et al:: Parasitic antigens alter macrophage polarization during Schistosoma japonicum infection in mice. Parasites \& Vectors 2014 7:122.

\section{Submit your next manuscript to BioMed Central and take full advantage of:}

- Convenient online submission

- Thorough peer review

- No space constraints or color figure charges

- Immediate publication on acceptance

- Inclusion in PubMed, CAS, Scopus and Google Scholar

- Research which is freely available for redistribution 